\title{
A Wolf Colony Search Algorithm Based on the Complex Method for Uninhabited Combat Air Vehicle Path Planning
}

\author{
Qiang Zhou ${ }^{1}$, Yongquan Zhou ${ }^{1,2}$ and Xin Chen ${ }^{1}$ \\ ${ }^{1}$ College of Information Science and Engineering, \\ Guangxi University for Nationalities, Nanning Guangxi 530006, China \\ ${ }^{2}$ Guangxi Key Laboratory of Hybrid Computation and IC Design Analysis, \\ Nanning Guangxi 530006, China
}

yongquanzhou@126.com

\begin{abstract}
Path planning for uninhabited combat air vehicle (UCAW is) a class of Complicated high dimensional optimization problem, which mainly centralizes on path planning considering the different kinds of constrains in the complex environment of wan In order to solve this problem, it is converted to a kind of constrained function optimization problem, and a wolf colony search algorithm based on the complex method is proposed, which combines the complex method with a wolf colony search algorithm, and it solves the problem of UCAV path planning successfully. The experiment results show that our proposed algorithm is feasible and effective to solve the problem of UCAKpath planing.
\end{abstract}

Keywords: Wolf colony search algorithm; eorplex method; UCAV path planning; constrained optimization

\section{Introduction}

Path planning is a new generation of low altitude penetration technology to achieve the purpose of terrain-following, terrain avoidance and flight with evading threat. While the path planning for UCAV is an important part in the mission planning system. The goal for path planning is to calculate the optimal or sub-optimal flight route for UCAV within the appropriate time so that the UCAV can break through the enemy threat environments and ensure the mission to conduct smoothly. UCAV path planning problem is a kind optimization problem which is related to the national defense and security, so a series of algorithms have been proposed to solve this complicated multiconstrained optimization problem, such as differential evolution (EA) [1, 13], genetic algorithm (GA) [2], ant colony optimization algorithm (ACO) [3], particle swarm optrimization (PSO) [4] and artificial bee colony (ABC) [19] and so on. However, these algorithms can hardly solve the contradiction between the global optimization and excessive information.

The wolf colony algorithm (WCA) is proposed by C. G Yang et al., in 2007. The algorithm is a swarm intelligence algorithm to simulate the intelligent predatory behaviors of the wolf colony. The wolf is a very intelligent animal. They are not alone when they catch and feed on food but by teams composed of several wolves. The wolf colony sends a few wolves to search quarry by smell. When the searching wolves discover the quarry, they notify the position of the quarry to the other wolves by howl. 
The other wolves get close to the quarry and besiege it. After they get the quarry, they distribute the food according to the strength of the wolf. At last, the weak wolves will be eliminated. WCA is proposed by this predatory behaviors of the wolf colony mixed with $\mathrm{ABC}$ successfully and applied in the path planning for the mobile robot. WCA has a good convergence rate, but solution accuracy is not high and easy to fall into local optimization.

In this paper, on the basis of the basic wolves' algorithm, we introduce a complex method strategy, and propose a wolf colony search algorithm based on the complex method (CWCA), and apply it in UCAV path planning problem successfully. Finally comparative experiments are conducted with ACO, BBO [15, 18], DE, ES [16], GA [8-9], PBIL [14], PSO, SGA [12], FA [5] and MFA [6] Experiment simulation results show that CWCA is more effective to solve UCAV path planning problem than other algorithms.

\section{UCAV Path Planning Mathematical Model}

\subsection{Problem description [20]}

Path planning for UCAV is formulated according to the practical situation and marks out the optimal flight route meeting certain performance requirements according to some performance indicators, and needs to consider many factors, such as the terrain, data, threat information, and time and so on. In order to solve UCAV patin planning problem, this paper builds a function optimization problem, ereates a mathematical model according to constraints, and finds the optimal flight routes satisfying the requirements. Shown as Figure 1, the actual problem is transformed into $(D$-dimensional function optimization problem by converting coordinates.

In Figure 1, we transform the onginal coordinate system into the new coordinate whose abscissa is the connection line from starting point to target point according to transform expressions shown in Equation (1) and (2), where, $(x, y)$ is a point in the original coordinate system, the point $\left(x^{\prime}, y^{\prime}\right)$ is a coordinate in the new rotating coordinate $O_{x^{\prime} y^{\prime}}, \theta$ is the rotation angle of the coordinate system.

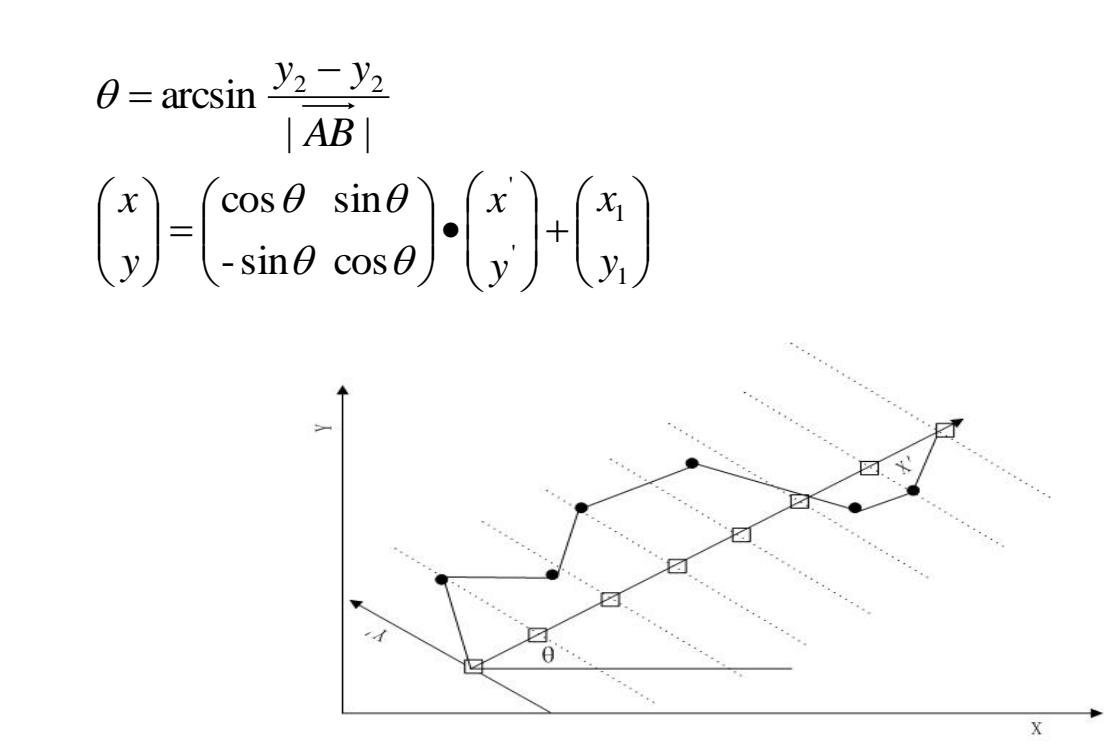

Figure 1. Coordinates Transformation Relation 
After the above conversion, we divide the abscissa of new coordinate $O_{x^{\prime} y^{\prime}}$, into $D$ equal partitions, and then get a vertical line and the coordinate of ordinate $Y^{\prime}$ for the corresponding node, so we can obtain a two-dimensional coordinate points set formed by $D$ points. The abscissas of these points are divided into equal partitions, so it is easy to get their point coordinate. We can get a path from start point to end point through connecting these points together, so UCAV problem is transformed into a constrained function optimization problem to let the problem become simplistic.

\subsection{Performance indicator}

In the problem description, UCAV needs to consider many factors to complete the task; these factors are performance indicators of the problem including safety perfornance indicator and performance indicator. UCAV needs to avoid some threats and pitfalls to make UCAV's threat minimum, and so does the fuel cost. We call it threat cost

Minimum of performance indicator for threat is calcuated by Equation (3).

$$
\min J_{t}=\int_{0}^{L} \omega_{t} d l
$$

Minimum of performance indicator for fuel is calculated by Equation (4).

$$
\min J_{f}=\int_{0}^{L} \omega_{f} d l
$$

Then the total performance indicatorsfor UCA V route are calculated by Equation (5).

$$
\min J=k J_{t}+(1-k) J
$$

where $J_{t}, J_{f}, J$ are the performance indjeator of threat, fuel and the total performance indicators for UCAV routerespectiyely, $\omega_{t}$ is the threat cost of each point on the route; $\omega_{f}$ is fuel cost of each point on the path; $L$ is the path length; $k, k \in[0,1]$ is balanced coefficient between safety performance and fuel performance, whose value is determined by the actual situation of the task UCAV performing, and depending on whether the aircraft is the emphasis on safety or on the time of task completion. The part is paid more attention; the share of the coefficient values will be greater.

\subsection{Threat cost}

When the UCAV is performing tasks, flying along the path $L_{i, j}$, the total threat cost generated by $N_{t}$ threats is calculated by Equation (6).

$$
\omega_{t, L_{i j}}=\int_{0}^{L_{i j}} \sum_{k=1}^{N_{t}} \frac{t_{k}}{\left[\left(x-x_{k}\right)^{2}+\left(y-y_{k}\right)^{2}\right]^{2}} d l
$$




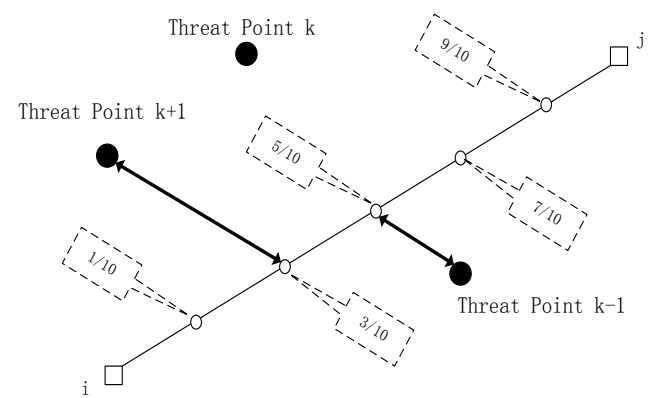

Figure 2. Calculation for Threat Cost

To simplify the calculations, we fetch a number of points of each segment to calculate threat cost. Shown in Figure 2, each edge is divided into five equal partitions, and the hreat cost on this edge is calculated by the five points. If the distance from the threat point to the edge is less than the threat radius, we can calculate the responding threat cost according to Equation (7).

$$
\omega_{t, L_{i j}}=\frac{L_{i j}^{5}}{5} \sum_{k=1}^{N_{t}} t_{k}\left(\frac{1}{d_{0.1, k}^{4}}+\frac{1}{d_{0.3, k}^{4}}+\frac{1}{d_{0.5, k}^{4}}+\frac{1}{d_{0.7, k}^{4}}+\frac{1}{d_{0.9, k}^{4}}\right)
$$

where $L_{i, j}$ is the length of the sub-segment connecting node $i$ and node $J ; d_{0,1, k}$ is the distance from the $1 / 10$ point on the sub-segment $L_{i, j}$ to the $k$-th threat; $t_{k}$ is threat level of the $k$-th threat.

\subsection{Fuel cost}

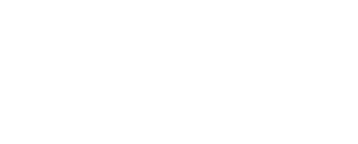

In the practical problem of UCAV path planning, fuel cost depends on path length. And the fuel cost of each poinf in path $\omega_{f}$ identically equal to 1 , so $\omega_{f}=L$, for simplicity, and fúl cost of each edge can be expressed by $\omega_{f, L_{i j}}=L_{i, j}$.

\section{CWCA}

In 1965,Box used simplex method [11] for solving unconstrained optimization problems to solve the constrained optimization problems, and the formed complex method for solving unconstrained optimization problems. In order to increase the optimization capability of WCA, the most strong wolves were selected to build a complex the complex centroid was made use of to produce a new point by reflecting, extension and shrinking, and the new point replaced bad points to continuously close to the optimal point. This algorithm is called CWCA.

\subsection{Initialize the wolf colony}

At this phase, the purpose is to let every wolf uniformly distribute in the domain of definition of the objective function. The scale of the wolf colony is $N$, the search space dimension is $D$, the position of the $i$-th wolf is 


$$
\begin{aligned}
& X_{i}=\left(x_{i 1}, \ldots, x_{i d}, \ldots, x_{i D}\right), \quad(1 \leq i \leq N, 1 \leq d \leq D) \\
& x_{i d}=x_{\text {min }}+\operatorname{rand} *\left(x_{\max }-x_{\min }\right)
\end{aligned}
$$

where rand is a random number uniformly distributed in the range $[0,1], x_{\max }$ and $x_{\min }$ are upper and lower limits of search space.

\subsection{Search the quarry}

In order to search the quarry, the optimal (that the fitness value is best) $q$ wolves are selected as the searching wolf, $q$ wolves are searching in $h$ directions around themselves, the position of wolf is $P_{i}\left(p_{i 1}, \ldots, p_{i d}, \ldots, p_{i D}\right)$. If the current position of the searching wolf is $P_{0}$, $P_{1}$ is produced around the current position $P_{0}$.If $P_{1}$ is better than $P_{0}$, then $P_{1}$ is set as the current position, and continue to search. When the searching number is largerthan max $d h$ or the current position is better than the searching position, the searching behavior ends.

In the $h$ positions produced around the searching wolf, $y_{j d}(1 \leq j \leq h$ is the position of $j$-th point of the $d$-th dimension, it can be calculated as follows:

$$
y_{j d}=x x_{i d}+\text { rand } * \text { stepa }
$$

Where, rand is a random number unifomly distributed in the region $[-1,1], x x_{i d}$ is the position of the $i$-th searching wolf of the $d$-th dimension. stepa is the searching step.

\subsection{Besiege the quarry}

Suppose that the position of the quary is the position of the searching wolf. When the searching wolves discoler the quarry they notify the position of the quarry to the other wolves by how. The other wolves get close to the quarry and besiege it. Then the updated position $z_{i d}$ of the $i$-th wolf of the $d$-th dimension is:

$$
z_{i d}=x_{i d}+\operatorname{rand}^{*} s_{\text {epp }} *\left(x_{l d}-x_{i d}\right)
$$

Where $x_{i d}$ is the carrebt position of the $i$-th wolf of the $d$-th dimension, rand is a random number uniformly distributed in the region $[0,1]$, stepb is movement step, $x_{i d}$ is the position of the $d$-th dimension of the quarry.

\subsection{Lead the wolf colony to search the quarry based on complex method}

in the wolf colony, we select $u$ strong wolves to build a complex, suppose the position of the $u$-th wolf is sorted as $\left(Z_{1}, Z_{2}, \ldots, Z_{u}\right)$ by fitness values. The complex steps are as follows:

Step1: calculate the complex centroid $Z_{c}$

$$
Z_{c}=\frac{1}{u-1} \sum_{i=1}^{u-1} Z_{i}
$$


Step2: calculate the reflection point $Z_{r}$

$$
Z_{r}=Z_{c}+\alpha\left(Z_{c}-Z_{u}\right)
$$

where $\alpha$ is reflection coefficient, generally taken $\alpha=1.3$. If $Z_{r}$ is better than $Z_{u}$, then $Z_{r}$ will replace $Z_{u}$; otherwise, turn to $S$ tep4.

Step3: extended operation

$$
Z_{e}=Z_{r}+\beta\left(Z_{r}-Z_{c}\right)
$$

Where $\beta$ is extension coefficient, taken $\beta=0.5 \sim 0.8$. If $Z_{e}$ is better than $Z_{u}$, then $Z_{e}$ will replace $Z_{u}$; otherwise, turn to $\mathbf{S t e p 4}$.

Step4: shrinked operation

$$
Z_{s}=Z_{u}+\chi\left(Z_{c}-Z_{u}\right)
$$

Where $\chi$ is shrinkage coefficient, taken $\chi=0.7$. If $Z_{s}$ is better than $Z_{u}$, then $Z_{s}$ will replace $Z_{u}$; otherwise, turn to $\mathbf{S t e p 1 .}$

A complex is constructed by selecting the strong wolves and conducts the wolf colony to search quarry so that the optimization capability of the algorithm 1 s improved and uneasy into local optimization.

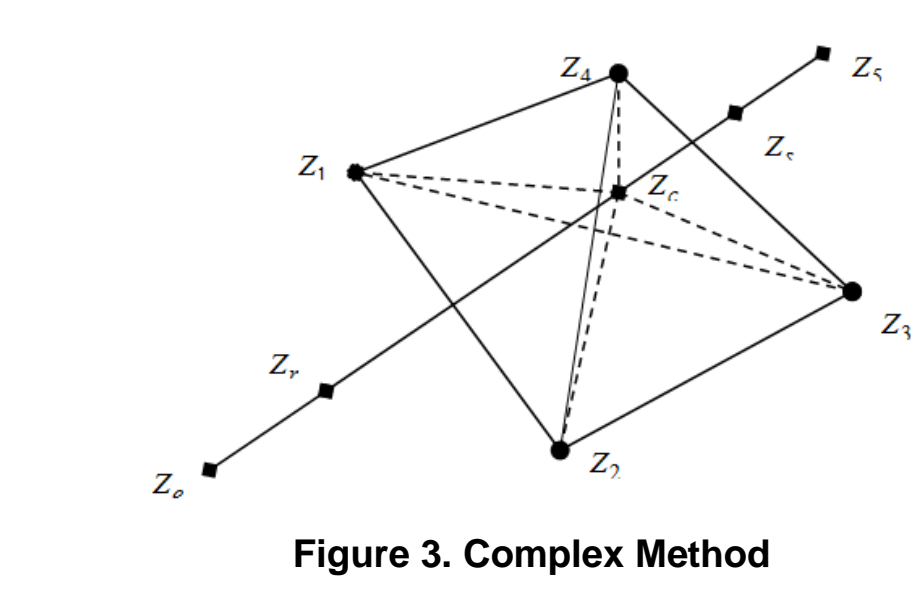

Figure 3 is an actualmodel of complex, the model is built by $Z_{1}, Z_{2}, Z_{3}, Z_{4}$, and the centroid $Z_{c}$ is found by the four points, and then $Z_{c}$ maps out reflection point $Z_{r}$ and shrinkage point $Z_{s}$ of $Z_{5}$. And the $Z_{r}$ that meets the conditions can get its extension point $Z_{e}$. This paper applies the strategy to WCA to search the optimal solution. Every point is the position of the wolf in the strategy

\subsection{Update the wolf colony}

The assignment rule of the wolf colony is to assign the food to the strong wolf at first and then to the weak one. The rule makes that the strong wolf gets enough food while the weak one gets little so that the weak wolf will starve to death. But the rule can ensure that the strong wolves prey next time, so the adapt ability of the wolf colony can be enhanced. By simulating the principle of survival of the fittest, the paper removes the worst $m$ artificial wolves in the colony and generates $m$ wolves randomly. Therefore, the algorithm can avoid the local optimum and the wolf colony becomes various. 


\subsection{The pseudo code of CWCA}

Five iterations are processed to build complex in the experiment, in order to explain the process of CWCA, the pseudo code of CWCA is given as follows:

\section{1: BEGIN}

2: Initialize: initialize the wolf colony randomly;

3: While (termination condition is not satisfied)

4: Select $q$ searching wolves by calculating fitness value $f(x)$;

5: The position of the strongest wolf is as the position of the quarry, other wolves besiege it;

6: Select $u$ better wolves after the colony sorted;

7: Number $=0$;

8 While $($ Number $<5)$

9: The complex is built by the position of $u$ wolves, the centroid of complex are calculated;

10: $x_{c}=\frac{1}{u-1} \sum_{i=1}^{u-1} x_{i}$;

11: Calculate reflection point, $x_{r}=x_{c}+\alpha\left(x_{c}-x_{u}\right)$

12: If $f\left(x_{r}\right)$ is better than $f\left(x_{u}\right)$

13: $x_{u}=x_{r}$;

14: Extended operation, $x_{e}=x_{r}+\beta\left(x-x_{c}\right)$;

15: If $f\left(x_{e}\right)$ is better than $f(x)$

16: $x_{u}=x_{e}$

17: Else

18: Shrinked operation $\chi_{s}=x_{u}+\chi\left(x_{c}-x_{u}\right)$

19: If $f\left(x_{s}\right)$ is better than $f\left(x_{u}\right)$

20: $x_{u}=x_{s}$

21: End If

22: End If

23: Else

24: Shrinked operation, $x_{s}=x_{u}+\chi\left(x_{c}-x_{u}\right)$;

25: If $f\left(x_{s}\right)$ is better than $f\left(x_{u}\right)$

26:

27: End If

End If

29: End While

30: Assign the food and update the wolf colony, remove the worst $m$ wolves and generate $m$ wolves randomly;

31: End While

32: Output the optimal individuals and fitness value of the populations;

33: End 


\section{CWCA for Solving UCAV Path Planning}

CWCA can adapt to the demand for UCAV path planning, and has a good search capability and improves the accuracy of the solution. Then we introduce the process of CWCA to apply in UCAV path planning.

\subsection{The steps of UCAV path planning based on CWCA}

Step1: Initialization. Initial the wolves' population size $n$, maximum number of iterations max $t$, searching step stepa, besieging step stepb and other parameters.

Step2: Transform coordinate system. A new coordinate is built in the original coordinate system. The abscissa is divided into equal $D$ partitions, and every aliquot ordinate is become the position of every dimension of wolves so the position of the $i$-th wolf is $\left(x_{i 1,} x_{i 2}, \ldots, x_{i D}\right)$.

Step3: Calculate the fitness value. Threat cost of every wolf is calculated by equation (5).

Step4: Searching the quarry. $q$ wolves are selected, namely the threat cost is optimal, searching the quarry according to equation (9).

Step5: Besiege the quarry, the position of the wolf Colony is updated by equation (10).

Step6: Build a complex. Select $w$ wolves which have better threat cost to build a complex by 3.4.

Step7: Update the wolfcolony. The papen removes the worst $m$ wolves in the colony and generates $m$ wolves randomly.

Step8: After completing an iteration, the algorithm enters the next iteration, and determines whether it meets the conditions or not. If it meets the conditions, it exits the loop, and records the position of welyes and indexes; otherwise go to Step2.

Step9: Inversely gansform the coordinates. Transform the new coordinates into the original coordinates, and record results. 


\subsection{The flow chart of UCAV path planning based on CWCA}

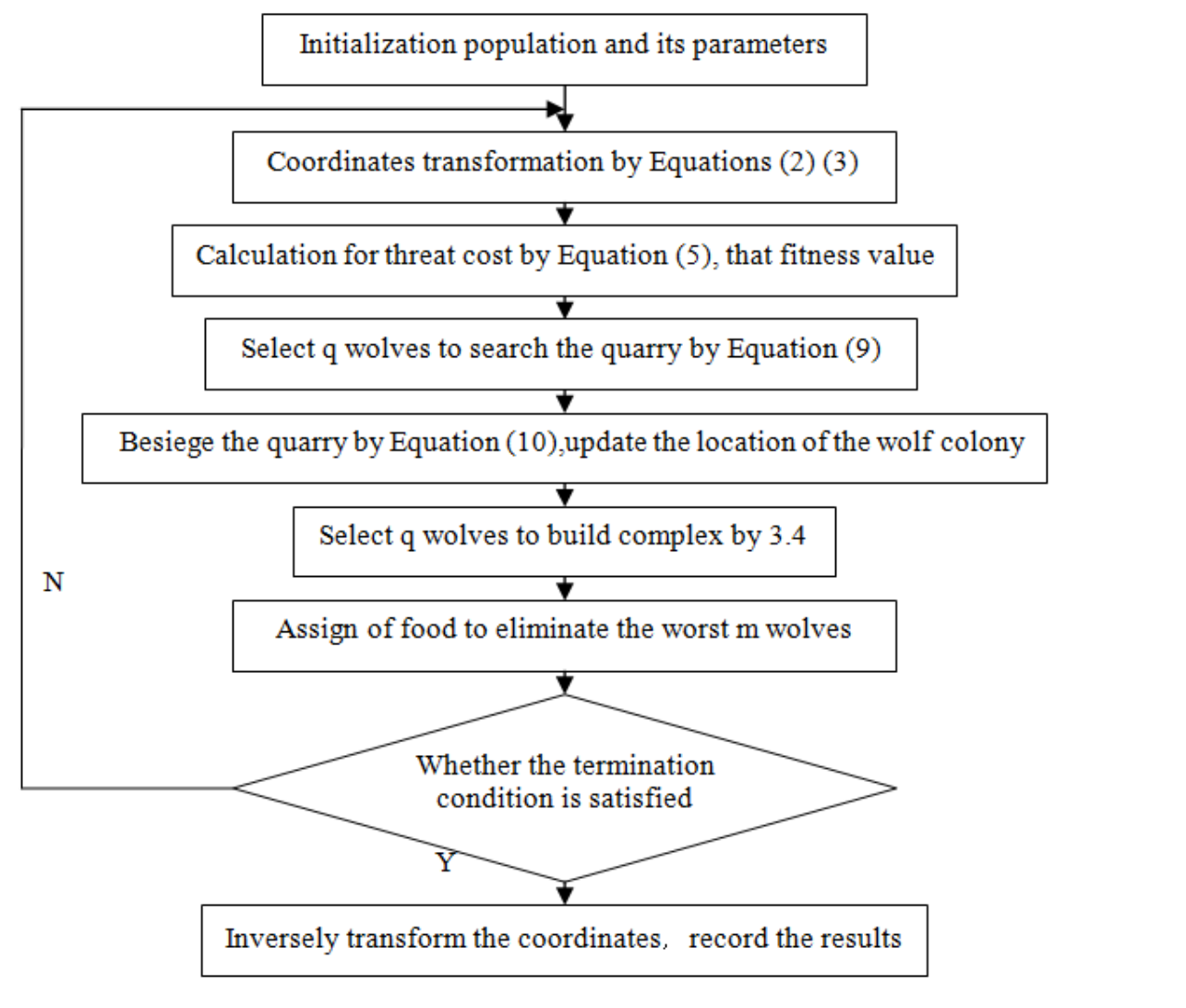

Figure 4. The Flow Chart of CWCA Solving UCAV Path Planning Problem

\section{Experiment Simulations}

This work use the emulator which is written by MATLAB 2010b and it is performed on the PC with Windows 7 OS, AMD athlon 640 Quad-core processors and 3GB memory.

In [10] an example is simulated. UCAV flight starts at the point $(10,10)$ and end at point $(55,100)$. There are five threats in this example, and they have their own threat grade and threat radius. Among, we set balanced coefficient between safety performance and fuel performance $k=0.5$. Information about known threats is shown Table 1.

\section{Table 1. Information about known threats}

\begin{tabular}{|l|c|c|c|c|c|}
\hline Cocation & {$[45,50]$} & {$[12,40]$} & {$[32,68]$} & {$[36,26]$} & {$[58,80]$} \\
\hline Threat radius & 10 & 10 & 8 & 12 & 9 \\
\hline Threat grade & 2 & 10 & 1 & 2 & 3 \\
\hline
\end{tabular}




\subsection{Influence of control parameters}

The choice of the control parameters has a great impact on different problems, the choice of besieging step stepb, extension coefficient $\beta$, and searching step stepa have different influences on route planning.

Order $D=20$, iterations $\max t=200$, , population size $n=30$, the number of searching wolves $q=5$, searching direction $h=4$, the maximum number of searching $\max d h=15$, searching step stepa $=0.5$, the number of worst wolves $m=5$. Each algorithm runs 100 times independently, best and means normalized optimization results on UCAV path planning problem are shown in Table 2. In order to represent the total performance value conveniently in the table, the threat cost all subtract 50 from the actual value, i.e., if a value is 0.5762 in the following table, then its corresponding value 50.5762 is its true value.

From the Table 2, when besieging step is stepb $=0.1$ Regardes of extension coefficient $\beta$ changing, the result exist only a little difference, here set $\beta=0.8$, because its mean value is the smallest.

Table 2. Best and mean normalize doptimization results on UCAV path planning problem on differentstepb and $\beta$

\begin{tabular}{|c|c|c|c|}
\hline Stepb & $\beta$ & Optimal & Mean \\
\hline 0.1 & 0.5 & 0.4191 & 0.5136 \\
\hline 0.3 & 0.5 & 0.4594 & 0.6241 \\
\hline 0.5 & 0.5 & 0.4467 & 0.7402 \\
\hline 0.1 & 0.65 & 0.4151 & 0.5044 \\
\hline 0.3 & 0.65 & 0.4558 & 0.6297 \\
\hline 0.5 & 00 & 0.4666 & 0.8785 \\
\hline 0.1 & 0.8 & 0.4173 & 0.5038 \\
\hline 0.3 & 0.8 & 0.4240 & 0.5788 \\
\hline 0.5 & 0.8 & 0.4367 & 0.6328 \\
\hline
\end{tabular}

Before besieging the quarry, the wolf colony searches the quarry, so the convergence speed of the argorithm is improved. But if the convergence speed is too fast, the algorithm is easy to fall into local optimization. So we simulate with different searching step stepa to find out the appropriate search step by comparing the total performance. From Table 2, we can know that the appropriate value for CWCA is $s t e p b=0.1, \beta=0.8$. Best and mean normalized optimization results on UCAV path planning problem on different searching step are shown in Table 3. 
Table 3. Best and mean normalized optimization results on UCAV path planning problem on different stepa

\begin{tabular}{|c|c|c|}
\hline Stepa & Optimal & Mean \\
\hline 0.05 & 0.3895 & $\mathbf{0 . 4 3 4 4}$ \\
\hline 0.08 & $\mathbf{0 . 3 9 0 1}$ & $\mathbf{0 . 4 3 4 4}$ \\
\hline 0.1 & 0.3926 & 0.4468 \\
\hline 0.3 & 0.4005 & 0.4550 \\
\hline 0.5 & 0.4173 & 0.5038 \\
\hline 1.0 & 0.4276 & 0.6208 \\
\hline 1.5 & 0.4517 & 0.6052 \\
\hline
\end{tabular}

From Table 3, when the searching step is stepa $=0.05$, the value is optimal but the running time is too long, and convergence speed is too slow. While the searching step meets an equation stepa $=0.1$, the result is worse than stepa $=0.05$, so set stepa $=0.08$, as the most appropriate, regardless of the convergence speed, the running time, and the accuracy of the solution are appropriate

\subsection{Effect of Dimensionality $D$}

In order to investigate the influence of the dimension on the performance of CWCA, this paper sets $d=5,10,15,20,25,30,35$,40 Eight different dimensions run 100 times independently, and compare with otherens of algortbm. Table 4, Table 5 and Table 6 show the best minima, the worst minima and the mean minima found by each algorithm respectively. In the simulations, we use the same population size and the maximum number of iterations $(n=30$, $\max t=200)$

From Table 4 we can see hat CWCA better than other algorithms no matter how large $D$ is. For example, $D=10$, the result got by CWCA is 0.6518 , but the solution of other algorithms is more than the accuracy of CWCA is higher than several other algorithms.

Table 4. Best normalized optimization results on UCAV path planning problem on different $D$

\begin{tabular}{|c|c|c|c|c|c|c|c|c|c|c|c|}
\hline \multirow{2}{*}{$D$} & \multicolumn{10}{|c|}{ Algorithm } \\
\cline { 2 - 15 } & ACO & BBO & DE & ES & FA & GA & MFA & PBIL & PSO & SGA & CWCA \\
\hline 5 & 11.3724 & 10.5302 & 4.3568 & 9.5895 & 4.3585 & 5.2471 & 4.3573 & 9.7627 & 5.1667 & 5.6538 & $\mathbf{3 . 3 7 9 1}$ \\
\hline 10 & 10.2281 & 2.942 & 1.3950 & 7.4272 & 1.3990 & 1.6068 & 1.3966 & 33.1123 & 2.2073 & 1.5489 & $\mathbf{0 . 6 5 1 8}$ \\
\hline 15 & 8.5298 & 2.5569 & 0.6114 & 8.2547 & 0.6172 & 0.8711 & 0.6115 & 57.2225 & 2.0969 & 0.8071 & $\mathbf{0 . 4 2 3 0}$ \\
\hline 20 & 10.4451 & 4.7230 & 0.5102 & 10.2329 & 0.4626 & 0.8252 & 0.4552 & 80.1521 & 2.4643 & 0.8460 & $\mathbf{0 . 3 9 0 1}$ \\
\hline 25 & 115490 & 5.5286 & 0.5512 & 13.3685 & 0.4908 & 1.2421 & 0.4571 & 109.7418 & 3.7378 & 1.2394 & $\mathbf{0 . 3 8 8 1}$ \\
\hline $\mathbf{3 0}$ & 13.2299 & 6.6071 & 0.8987 & 15.7251 & 0.6828 & 1.9218 & 0.5160 & 180.1498 & 3.2993 & 1.6165 & $\mathbf{0 . 3 9 1 3}$ \\
\hline 35 & 16.9599 & 13.0206 & 2.5372 & 16.7445 & 1.0829 & 2.3109 & 0.4709 & 220.3331 & 5.5025 & 1.6326 & $\mathbf{0 . 3 7 9 3}$ \\
\hline 40 & 19.7946 & 13.5504 & 4.5490 & 18.2314 & 1.5225 & 2.2084 & 0.4506 & 340.6174 & 5.7367 & 2.6180 & $\mathbf{0 . 3 6 3 8}$ \\
\hline
\end{tabular}

Table 5 shows the worst normalized optimization results on different $D$. From it, we can see that PBIL gets the worst results compared with other algorithms, its results increase with $D$ rising. When $D$ increases from 25 to 30 , the results increase in a nearly four-fold. While the result obtained by CWCA decreases by 0.3 . With the rise of the dimension, results obtained by most of algorithms are in a rising trend, but some parts of algorithms decline 
when $D$ increases from 20 to35. The worst result of CWCA is better than several other algorithms.

\section{Table 5. Worst normalized optimization results on UCAV path planning problem on different $D$}

\begin{tabular}{|c|c|c|c|c|c|c|c|c|c|c|c|}
\hline \multirow{2}{*}{$D$} & \multicolumn{10}{|c|}{ Algorithm } \\
\cline { 2 - 14 } & ACO & BBO & DE & ES & FA & GA & MFA & PBIL & PSO & SGA & CWCA \\
\hline 5 & 13.3199 & 121.5724 & 12.2083 & 62.2665 & 15.7395 & 11.6013 & 12.4186 & 22.2463 & 16.0713 & 11.2006 & $\mathbf{3 . 5 3 0 7}$ \\
\hline 10 & 18.1911 & 26.8270 & 6.7358 & 73.4605 & 6.7095 & 10.1096 & 3.7858 & 69.2468 & 18.6221 & 6.1652 & $\mathbf{0 . 7 0 9 2}$ \\
\hline 15 & 11.0084 & 40.3705 & 12.5808 & 53.8683 & 44.2763 & 7.4472 & 3.8319 & 139.2557 & 37.3201 & 11.7962 & $\mathbf{0 . 4 4 7 9}$ \\
\hline 20 & 17.1887 & 28.2063 & 14.5783 & 31.4587 & 28.9142 & 9.1795 & 2.0279 & 287.3709 & 28.1596 & 18.9518 & $\mathbf{0 . 5 5 1 1}$ \\
\hline 25 & 12.0733 & 30.3315 & 19.6664 & 33.9148 & 16.4518 & 10.3977 & 3.7043 & 649.6845 & 28.1399 & 15.6967 & $\mathbf{0 . 5 2 2 1}$ \\
\hline 30 & 14.7139 & 28.5885 & 24.1279 & 41.3024 & 15.9757 & 12.7183 & 8.3364 & 2364.08 & 43.6950 & 14.7140 & $\mathbf{0 . 5 4 0 0}$ \\
\hline 35 & 18.7271 & 43.8512 & 34.4447 & 38.7646 & 33.8871 & 24.4790 & 5.8830 & 6312.96 & 32.8328 & 17.6058 & $\mathbf{0 . 5 5 4 0}$ \\
\hline 40 & 27.0641 & 40.7087 & 43.2604 & 46.4224 & 36.6626 & 22.0688 & 7.7236 & 7053.50 & 34.7302 & 17.8669 & $\mathbf{0 . 6 8 2 1}$ \\
\hline
\end{tabular}

Table 6 is the mean normalized optimization results on different $D$, when CWCA sets different $D$, the result of CWCA is better than otheragorithms. For instance, when $D$ meets the equation $D=40$, the mean of CWCA is 0.509 , while the mean of ACO is 24.5754. It can be seen clearly that CWCA is better than ACO, and the results of other algorithms are worse than CWCA.

Table 6. Mean normalized optimization results on UCAV path planning problem on different $D$

\begin{tabular}{|c|c|c|c|c|c|c|c|c|c|c|c|}
\hline \multirow{2}{*}{$D$} & \multicolumn{11}{|c|}{ Algorit } \\
\hline & $\mathrm{ACO}$ & BBO & $\mathrm{DE}$ & ES & FA & CA & MFA & PBIL & PSO & SGA & CWCA \\
\hline 5 & 11.5151 & 22.7318 & & 30.7228 & & 0.4747 & 9.1673 & 16.1391 & 9.9061 & $\begin{array}{c}10.501 \\
3\end{array}$ & 3.4072 \\
\hline 10 & 11.9485 & 7.9650 & & 26.2868 & 2.1801 & 2.5422 & 1.5740 & 51.4355 & 7.0411 & 2.2790 & 0.6819 \\
\hline 15 & 10.2554 & 9.5257 & 100 & 21.8618 & 2.8217 & 2.1880 & 0.8967 & 78.2477 & 8.3395 & 1.8910 & 0.4344 \\
\hline 20 & 16.2205 & 11.8761 & 2.7221 & 20.892 & 3.7327 & 3.0900 & 0.7004 & 135.4365 & 8.2483 & 3.1670 & 0.4344 \\
\hline 25 & 11.5674 & 147800 & 4.4081 & 22.7794 & 3.9039 & 3.7814 & 0.9987 & 207.7272 & 10.2627 & 4.1567 & 0.4458 \\
\hline 30 & 13.9593 & 17.8746 & 9.9884 & 24.7757 & 4.9621 & 5.0079 & 1.3568 & 345.5447 & 12.3847 & 4.5211 & 0.4339 \\
\hline 35 & 18.3108 & 21.5615 & 17.902 & 26.5217 & 5.9955 & 5.9599 & 1.6009 & 634.6550 & 14.1354 & 5.8260 & 0.4379 \\
\hline 40 & 24.5754 & 24.8531 & & 30.2595 & 7.8558 & 7.4927 & 2.1978 & 1119.72 & 14.8845 & 7.1100 & 0.5099 \\
\hline
\end{tabular}

From Figure 4 Figure 5, and Figure 6, it can be seen that the optimal value, the worst value and the average value obtained by CWCA are better than other algorithms. Though with the increase of dimension, the results of CWCA also increase, yet in some cases, it is in a downward rend and the accuracy of the solution is improved indeed. With the increasing of dimensions, the complexity and running time of the algorithm grow, and the accuracy is not improyed too much. In summary from these tables above, setting $D=20$ or 25 is more appropriate, the accuracy of the two solutions have some difference. Considering the complexity of the algorithm, if $D=20$, its complexity is $o(n)$, that when we set $D=25$, its complexity is $1.25 O(n)$, so we set $D=20$.

Figures 5 12 show that When $D$ is set different values; we can get the position of fight at every moment. In Figure 5, $D=5$, it can be seen that the fight route is consist of seven points, and after removing the start point and the end point, the route is just matched up to $D=5$. We can see the flight route and the threat center form the figure. We can also see how the airplane 
escapes from the threat center to make the threat cost become smallest. UCAV path planning is to find an appropriate path which the threat is smallest.

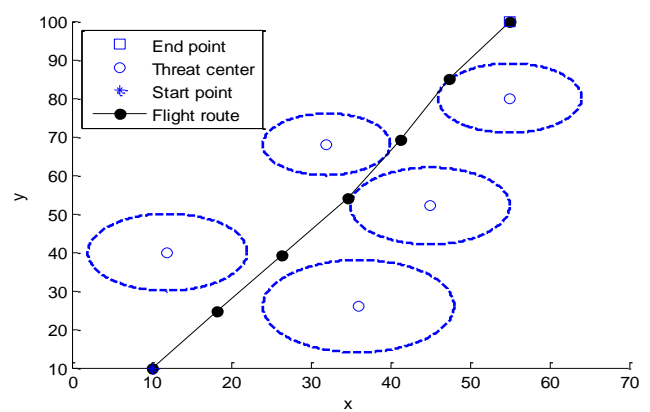

Figure 5. $D=5$ the result of Path Planning

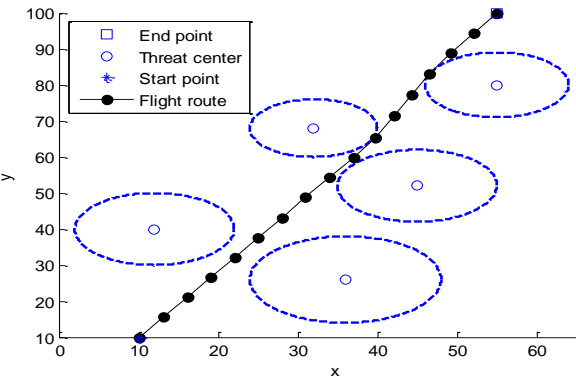

Figure 7. $D=15$ the result of Path Planning

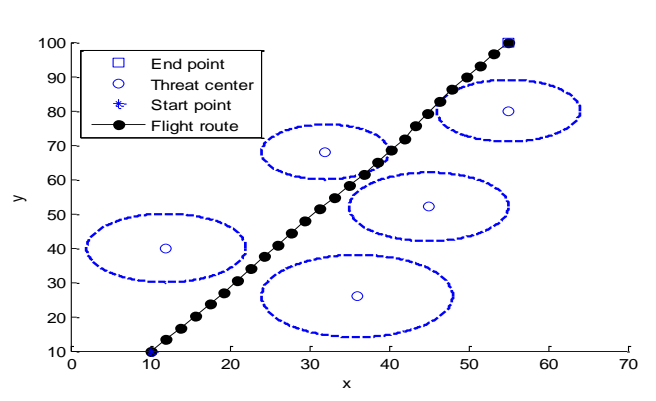

Figure 9. $D=25$ the result of Path Planning

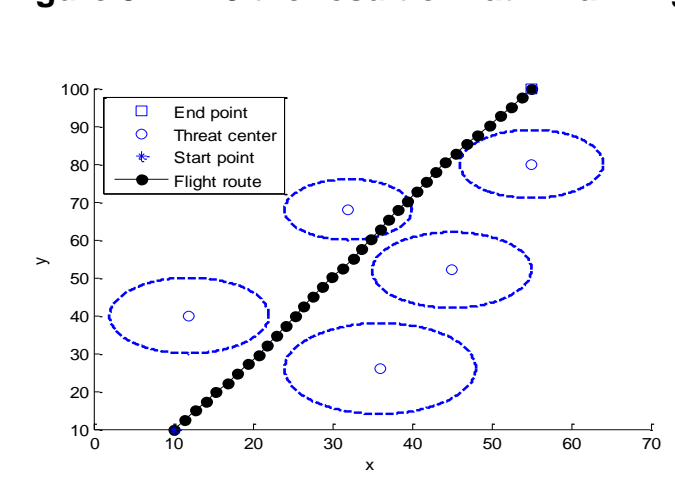

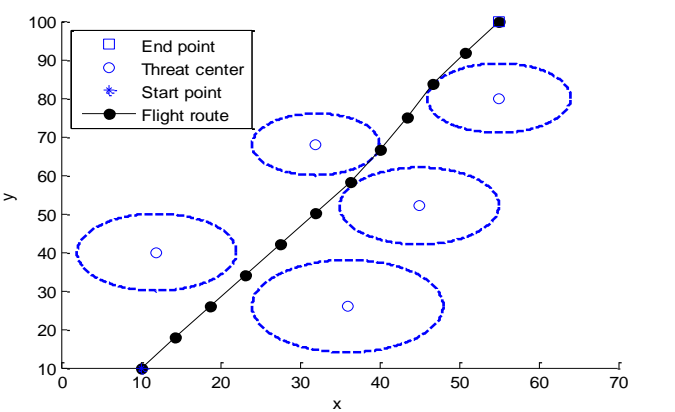

Figure 6. $D=10$ the result of Path Planning

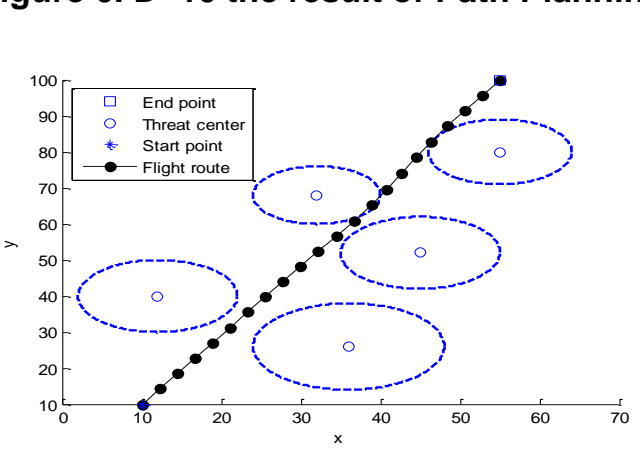

Figure 8. $D=20$ the result of Path Planning

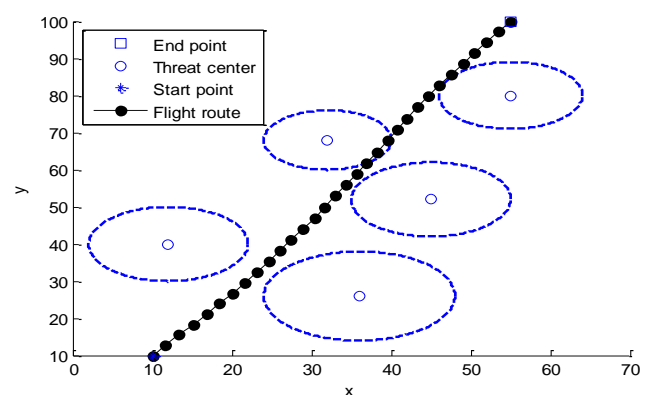

Figure 10. $D=30$ the result of Path Planning

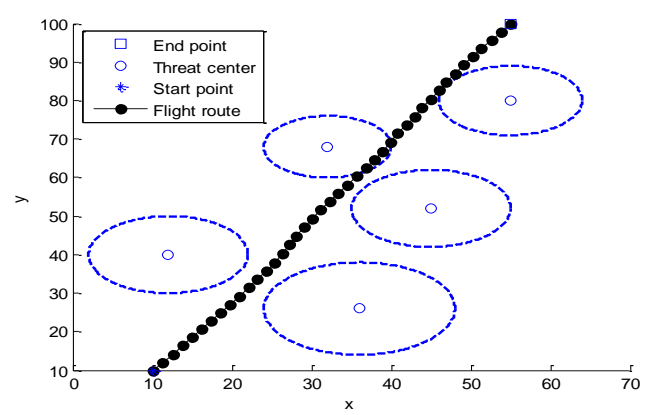

Figure 11. $D=35$ the result of Path Planning Figure 12. $D=40$ the result of Path Planning 


\subsection{Effect of maximum iterations max $t$}

The choice of the maximum number of iterations is of vital importance to solve the algorithm. The choice of the maximum number of iterations has a directly effect on the solution accuracy of different problems, so we set the maximum number of iterations as 50, $100,150,200,250$ respectively, and each algorithm runs 100 times independently ( $N=30, d=20$ ). Table 7, Table 8 and Table 9 are the best, worst and mean results respectively on different maximum number of iterations for every algorithm.

Table 7. Best normalized optimization results on UCAV path planning problem on different maxt

\begin{tabular}{|c|c|c|c|c|c|c|c|c|c|c|c|}
\hline \multirow{2}{*}{$\operatorname{maxt}$} & \multicolumn{10}{|c|}{ Algorithm } & \multirow{2}{*}{$\frac{\diamond}{\sqrt{C A}}$} \\
\hline & $\mathrm{ACO}$ & BBO & $\mathrm{DE}$ & ES & FA & GA & MFA & PBIL & PSO & SGA & \\
\hline 50 & 9.6276 & 4.8658 & 3.6814 & 7.7983 & 1.4713 & 1.6118 & 0.7030 & 66.0274 & 3.6012 & 1.70 & 0.4232 \\
\hline 100 & 11.5242 & 4.2616 & 0.9439 & 40.2329 & 0.6577 & 4.5294 & 0.5382 & 48.9269 & 2.3357 & 1.3520 & 0.3986 \\
\hline 150 & 5.6381 & 5.6105 & 0.7015 & 9.8027 & 0.5459 & 1.2042 & 0.4857 & 47.4630 & 2.61 & 0.9498 & 0.3956 \\
\hline 200 & 11.2445 & 2.9424 & 0.5188 & 10.7960 & 0.4931 & 1.0695 & 0.4 & 18.6980 & & 0.8392 & 0.3901 \\
\hline 250 & 9.7607 & 3.5209 & 0.4829 & 10.2540 & 0.4753 & 0.8781 & & 20.8802 & 2.9229 & 0.7839 & 0.3878 \\
\hline
\end{tabular}

Table 8. Worst normalized optimization results on UCAV path planning problem on different maxt

\begin{tabular}{|c|c|c|c|c|c|c|c|c|c|c|c|}
\hline $\operatorname{maxt}$ & \multicolumn{5}{|l|}{$\mathrm{ACO}$} & GA & MFA & PBIL & PSO & SGA & CWCA \\
\hline 50 & 18.7099 & 28.6806 & 31.3392 & 33.1927 & 28.0425 & 10.9773 & 4.6726 & 312.9370 & 33.1539 & 17.6318 & 0.6800 \\
\hline 100 & 17.7404 & 28.2427 & 19.4058 & 33.1979 & 29.3022 & 11.1678 & 4.5749 & 373.8334 & 27.8806 & 11.6446 & 0.5710 \\
\hline 150 & 17.4223 & 40.1797 & 14.5560 & 8.12 & 27.848 & 17.5637 & 4.9631 & 210.6140 & 28.3542 & 15.2145 & 0.5691 \\
\hline 200 & 17.0679 & 32.1981 & 16.6736 & 52.4090 & & 1.9124 & 9.1502 & 183.9630 & 28.2524 & 6.7380 & 0.5511 \\
\hline 250 & 17.0679 & 27.6544 & 8.5122 & 46.0828 & & 7.4338 & 3.6783 & 169.1446 & 29.6341 & 16.2672 & 0.5714 \\
\hline
\end{tabular}

Table 9. Mean normalized optimization results on UCAV path planning problem

\begin{tabular}{|c|c|c|c|c|c|c|c|c|c|c|c|}
\hline \multirow{2}{*}{$\max t$} & \multicolumn{11}{|c|}{ Algorithm } \\
\hline & $\mathrm{ACO}$ & $\mathrm{BBO}$ & DE & ES & FA & GA & MFA & PBIL & PSO & SGA & CWCA \\
\hline 50 & 16.2648 & 14.2076 & 132645 & 20.3152 & 6.2034 & 4.1142 & 1.9576 & 151.9844 & 9.9671 & 5.2389 & 0.5407 \\
\hline 100 & 16.3500 & 13.4074 & 7,3195 & 20.4387 & 4.3526 & 3.7731 & 1.3048 & 113.6434 & 8.9057 & 3.7475 & 0.4713 \\
\hline 150 & 16.1722 & 12.6978 & 3.5255 & 19.9485 & 4.1809 & 3.4671 & 0.9933 & 90.8722 & 8.5509 & 3.1680 & 0.4563 \\
\hline 200 & 16.2154 & 11.8556 & 2.3975 & 20.7501 & 2.2791 & 2.9711 & 0.8984 & 74.1964 & 8.9892 & 2.3792 & 0.4344 \\
\hline 250 & 16.044 & 11.9654 & 2.4849 & 20.1739 & 2.2064 & 2.6605 & 0.7025 & 65.4942 & 9.2143 & 2.5929 & 0.4347 \\
\hline
\end{tabular}

From Table 7, Table 8, and Table 9, it can be seen that the CWCA can get better results than other algorithms. With the rising of the number of iterations, the accuracy of the solution of every algorithm grows high. But the complexity of the algorithm is getting higher as the number of iterations increasing, and the accuracy of the algorithm is not improved greatly. Such as in Table 7 , when the maximum number of iterations is 250 , the solution of MFA increases about 0.01 compared in 200 iterations, while the algorithm iterates more for 50 times. But the accuracy of the solution of CWCA has little change, the maximum number of iterations is set 200, and CWCA is better than other algorithms when solving the UCAV path planning problem. 

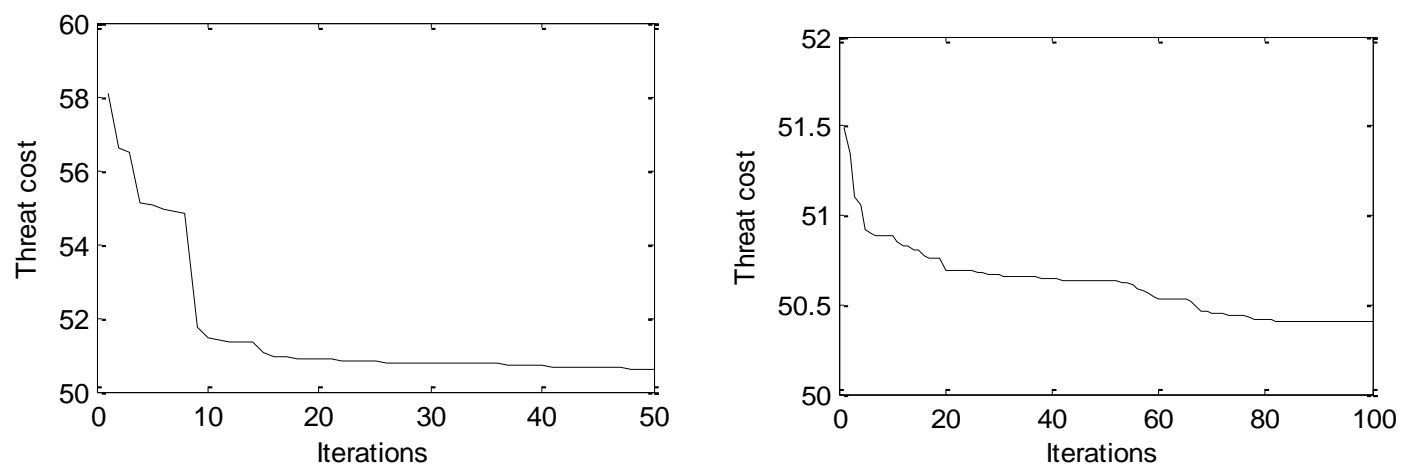

Figure. 13 Convergence curve of maxt=50

Figure. 14 Convergence curve of maxt $=100$
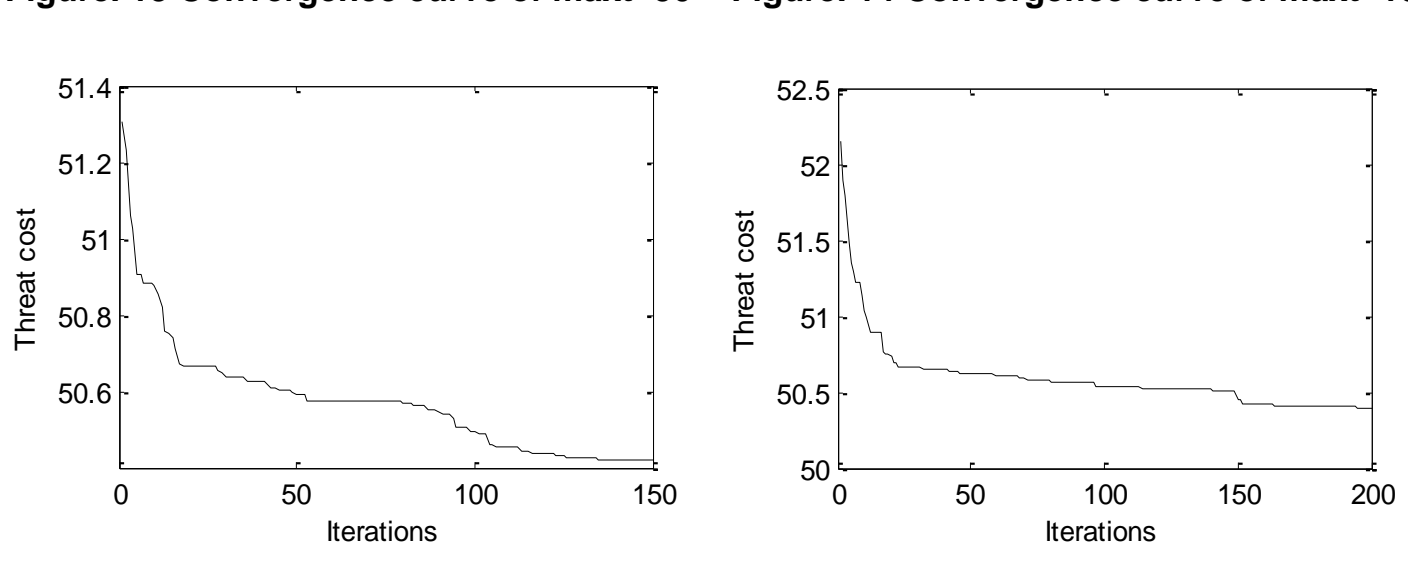

Figure. 15 Convergence curve of maxt=150 Figure. 16 Convergence curve of maxt=200

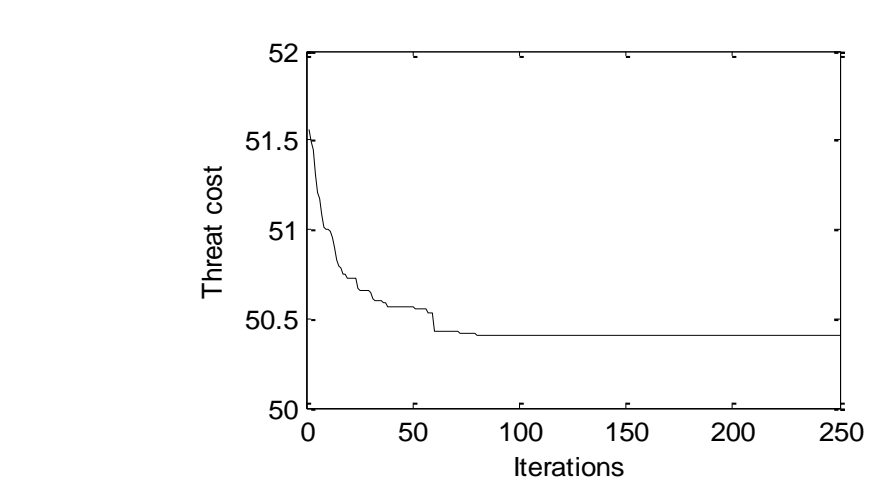

Figure. 17 Convergence curve of maxt=250

Pigure 13 to Figure 17 is convergence curve figures of CWCA on different maximum number of iterations. From the figures, we can see that the convergence speed of CWCA is fast, and it is not easy to fall into local optimization. Such as Figure 15, it is converged from 20-th iteration to 80-th iteration; the algorithm jump out of local optimization at last, the accuracy of the solution is improved. It is uneasy to precocious and is effective for solving UCAV path planning problem. 


\section{Conclusions}

For UCAV path planning problem, this paper proposes a wolf colony search algorithm based on the complex method, which let WCA mix with the complex method and the complex method as the wolf colony's leading strategy. The UCAV can find the safe path by connecting the chosen nodes while avoiding the threat areas and costing minimum fuel. Compared with other algorithms, experiment results show that CWCA is a more feasible and effective way in UCAV path planning.

\section{Acknowledgements}

This work is supported by National Science Foundation of China under Grant No. 61165015. Key Project of Guangxi Science Foundation under Grant No. 2012GXNSFDA053028, Key Rroject of Guangxi High School Science Foundation under Grant No. 20121ZD008, and the Innovation Project of Guangxi Graduate Education under Grant No. YCSZ2012063.

\section{References}

[1] R. Storn and K. Price, "Differential Evolution-A Simple and EfficientHeuristic for Global Optimization over Continuous Space”, Journal of Global Optimization, vol. 11, (1997), pp. $341-359$.

[2] J. H. Holland, "Adaptation in Natural and Artificial Systems", University of Mî́chigan Press, Ann Arbor, Mich, USA, (1975).

[3] W. Ye, D. W. Ma and H. D. Fan, "Algorithm for Low Altitude Penetration Aircraft Path Planning with Improved Ant Colony Algorithm", Chinese Journar of Aeronautics, vol. 18, no. 4, (2005), pp. 304-309.

[4] J. Kennedy and R. Eberhart, "Particle Swarm Optimization", Neural Network, Proceedings, IEEE International Conference on IEEE, vol. 4, (1995), pp. 1942-1948.

[5] X. S. Yang, "Nature-Inspired Metaheuristic Algorithms", Luniver Press, Frome, (2008).

[6] G. Wang, L. Guo, H. Duan, L. Liu and H Wang, "A Modified Firefly Algorithm for UCAV Path Planning", International Journal of Hybird information Technology, vol. 5, no. 3, (2012), pp. 123-143.

[7] C. Yang, X. Tu and J. Chen, "Algeri hm of Marriage in Honey Bees Optimization Based on the Wolf Pack Search", International Conference on Intelligent Pervasive Computing, (2007), pp. 462-467.

[8] Y. V. Pehlivanoglu, "A new/yibrational genetic algorithm enhanced with a Voronoi Diagram for Path Planning of Autonomous OAV", Aerospace Science and Technology, vol. 16, no. 1, (2012), pp.47-55.

[9] J. L. Yan, "Evolutionary Algorithm Based Route Planer for Unmanned Air Vehicles", A Thesis in Control Science and Engineerng, Nanjing University of Aeronautics and Astronautics Press, (2008).

[10] H. B. Duan, X Y Zhang and C. F. Xu Bio-Inspired Computing”, Science Press, Beijing, China, (2011).

[11] M. J. Box, "A New Method ofConstrained Optimization and A Comparison with Other Method", Computer Journal, vol 8, no. 1, (1965), pp-42-52.

[12] W. Khatib and P. J. Fleming, "The stud GA: A Mini Revolution? Parallel Problem Solving from NaturePPSN V”, Springer Berlin Heidelberg, (1998), pp. 683-691.

[13] Y. Zhou, Q. Lou, If. Chen and J. Wu, "Using Differential Evolution Invasive Weed Optimization for Solving Circle Packing Problem", Advanced Science Letters, vol. 19, no. 6, (2013), pp. 1807-1810.

[14] S. Baluja, "Population-based Incremental Learning", A Method for Integrating Genetic Search Based Function Optimization and Competitive Learning, Carnegie-Mellon Univ Pittsburgh Pa Dept. of Computer Science, (1994).

[15] D. Simon Biogeography-based Optimization", Evolutionary Computation, IEEE Transactions on, vol. 12, no 6, (2008), pp. 702-713.

[16] E. Mayr, "Behavior Programs and Evolutionary Strategies", Natural Selection Sometimes Favors a Genetically "closed" Behavior Program, Sometimes An "open" one, American Scientist, vol. 62, no. 6, (1974), pp. 650-659.

[17] C. Liu, X. Yan, C. Liu and H. Wu, "The Wolf Colony Algorithm and Its Application", Chinese Journal of Electronics, vol. 20, no. 2, (2011), pp. 212-216.

[18] D. Simon, "The Matlab Code of Biogeography-based Optimization", http://academic.csuohio.edu/simond/bbo/, (2012).

[19] C. Xu, H. Duan and F. Liu, "Chaotic Artificial Bee Colony Approach to Uninhabited Combat Air Vehicle (UCAV) Path Planning”, Aerospace Science and Technology, vol. 14, no. 8, (2010), pp. 535-541.

[20] H. Duan, X. Zhang and C. Xu, "Bio-inspired Computing”, Science Press, (2011), pp. 121-126. 


\section{Authors}

Qiang Zhou M.S. He is currently research interest is in computation intelligence, swarm intelligence algorithm.

Yongquan Zhou, Ph.D \& Prof. He received the MS degree in computer science from Lanzhou University, Lanzhou, China, in 1993 and the Ph.D degree in computation intelligence from the Xiandian University, Xi'an, China, in 2006. He is currently a professor in Guangxi University for Nationalities. His research interests include computation intelligence, neural networks, and intelligence information processing et al. He has published 1 book, and more than 150 research papers in journals.

Xin Chen M.S. He is currently research interest is in computation intelligence, swarm intelligence algorithm.

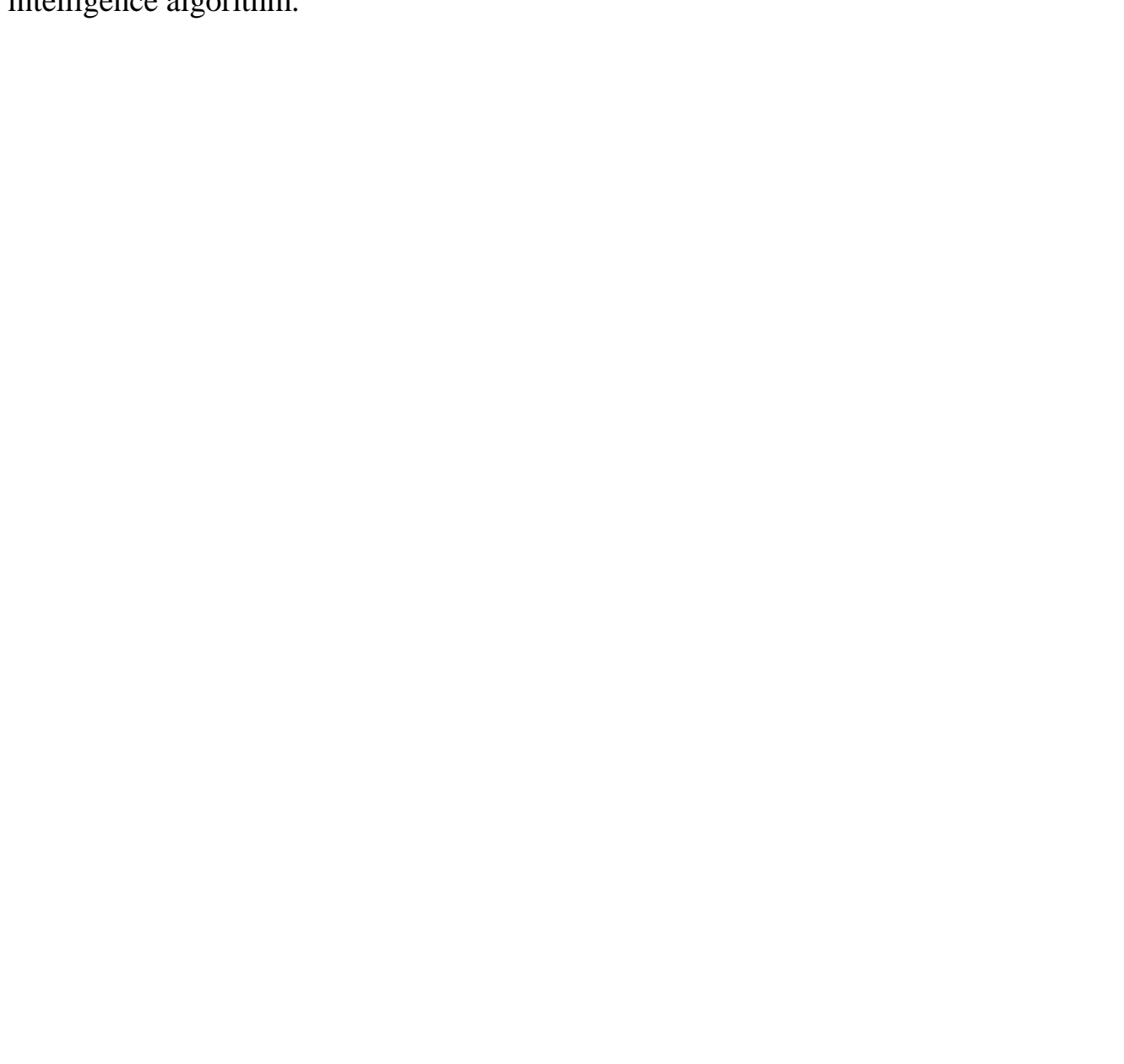


International Journal of Hybrid Information Technology

Vol.7, No.1 (2014)

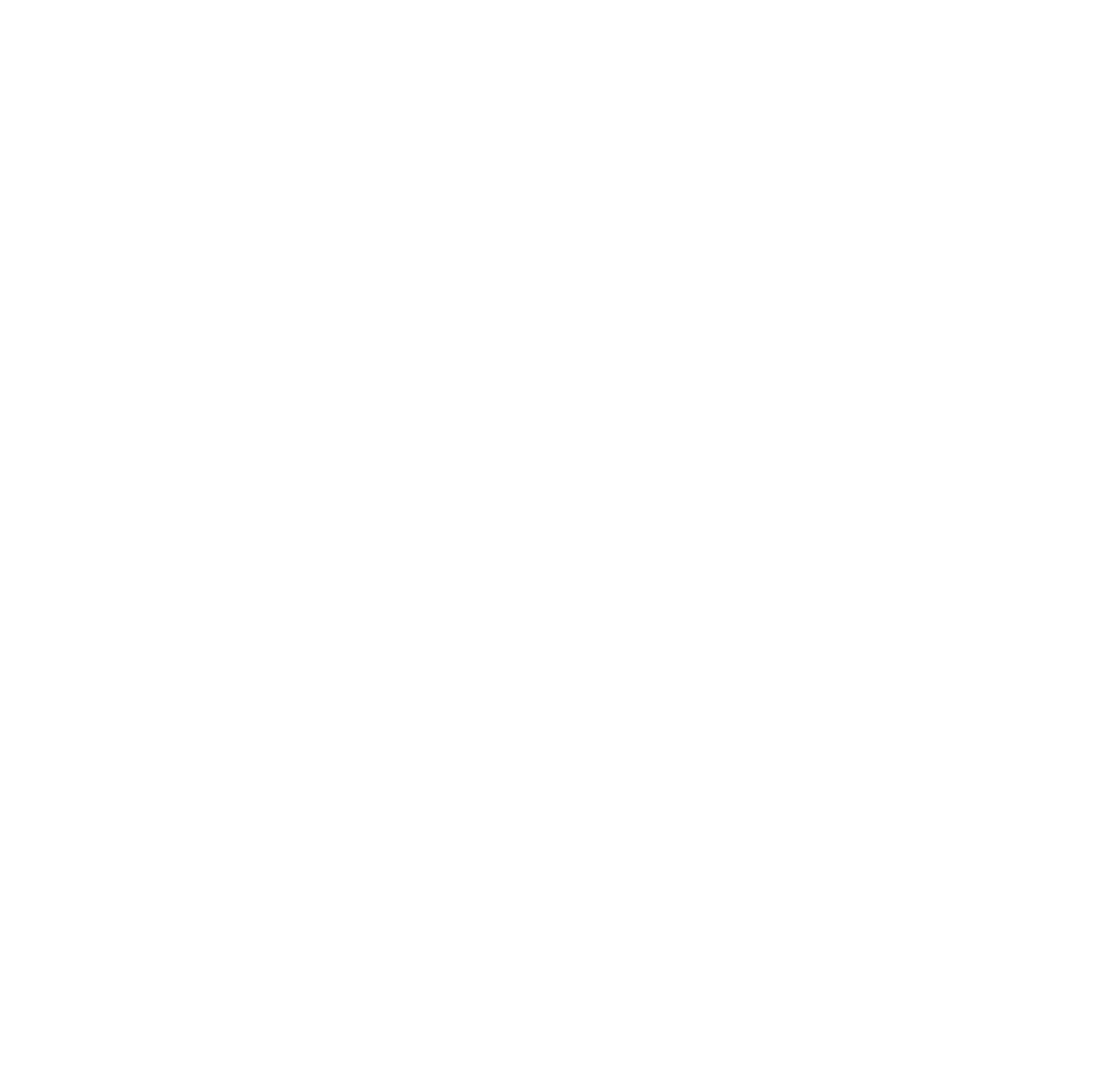

